\title{
CDX2 autoregulation in human intestinal metaplasia of the stomach: impact on the stability of the phenotype
}

\author{
Rita Barros, ${ }^{1}$ Luis Teixeira da Costa, ${ }^{1,2}$ João Pinto-de-Sousa, ${ }^{3,4}$ Isabelle Duluc, ${ }^{5}$ \\ Jean-Noel Freund, ${ }^{5}$ Leonor David, ${ }^{1,4}$ Raquel Almeida ${ }^{1,4}$
}

\begin{abstract}
- Additional data are published online only. To view these files please visit the journal online (http://gut.bmj.com).

1 Institute of Molecular Pathology and Immunology of the University of Porto, Porto, Portugal

${ }^{2}$ ICAAM, Universidade de Évora, Évora, Portugal

${ }^{3}$ Department of Surgery, Hospital S. João, Porto, Portugal

${ }^{4}$ Faculty of Medicine of the University of Porto, Porto, Portugal

Inserm U682, Strasbourg, France
\end{abstract}

\section{Correspondence to}

Raquel Almeida, IPATIMUP, Rua Dr Roberto Frias s/n, 4200-465 Porto, Portugal;

ralmeida@ipatimup.pt

Revised 8 October 2010 Accepted 11 October 2010 Published Online First

9 December 2010

\section{ABSTRACT}

Background and aims Intestinal metaplasia (IM) is a gastric preneoplastic lesion that appears following Helicobacter pylori infection and confers an increased risk for development of cancer. It is induced by gastric expression of the intestine-specific transcription factor CDX2. The regulatory mechanisms involved in triggering and maintaining gastric CDX2 expression have not been fully elucidated. The $\mathrm{Cdx} 2^{+/-}$mouse develops intestinal polyps with gastric differentiation and total loss of $\mathrm{Cdx} 2$ expression in the absence of structural loss of the second allele, suggesting a regulatory defect. This putative haplo-insufficiency, together with the apparent stability of IM, led to the hypothesis that CDX2 regulates its own expression through an autoregulatory loop in both contexts.

Methods Gastrointestinal cell lines were co-transfected with wild-type or mutated $\mathrm{Cdx} 2$ promoter constructs and CDX2 expression vector for luciferase assays.

Transfection experiments were also used to assess endogenous CDX2 autoregulation, evaluated by RT-PCR, qPCR and western blotting. Chromatin

immunoprecipitation was performed in a cell line, mouse ileum and human IM.

Results CDX2 binds to and transactivates its own promoter and positively regulates its expression in gastrointestinal human carcinoma cell lines. Furthermore, CDX2 is bound to its promoter in the mouse ileum and in human gastric $\mathrm{IM}$, providing a major contribution to understanding the relevance of this autoregulatory pathway in vivo.

Conclusion The results of this study demonstrate another layer of complexity in CDX2 regulation by an effective autoregulatory loop which may have a major impact on the stability of human IM, possibly resulting in the inevitable progression of the gastric carcinogenesis pathway.

\section{INTRODUCTION}

Intestinal metaplasia (IM) of the stomach is a preneoplastic lesion that confers an increased risk for the development of gastric carcinoma, which remains the second leading cause of cancer death worldwide. ${ }^{1}$ IM occurs most frequently in the gastric carcinogenic cascade following Helicobacter pylori infection, which leads to the appearance of a chronic gastritis, atrophy, progression to IM and, ultimately, gastric cancer. ${ }^{2}$ Eighty per cent of the gastric carcinomas appear in the context of $\mathrm{IM}^{3}$ and the presence of this preneoplastic lesion results in a 2-6-fold increased risk for subsequent cancer development. ${ }^{3-5}$ Furthermore,

\section{Significance of this study}

What is already known about this subject?

- The expression of the CDX2 gene does not occur in the normal stomach, but its ectopic gastric expression is necessary for intestinal metaplasia development.

- Regulatory mechanisms for CDX2 are still poorly understood and fail to explain the stability of the intestinal metaplasia phenotype.

- Mouse models have suggested a haplo-insufficiency for $\mathrm{Cdx} 2$ and in vitro studies have suggested a cell type-specific autoregulatory mechanism.

What are the new findings?

- CDX2 regulates its own promoter in gastric and intestinal cell lines, and active binding sites were identified in the proximal promoter.

- CDX2 regulates its own endogenous expression in different cellular contexts, initiating its own expression.

- CDX2 is bound to its own promoter in a gastric cell line, in the mouse ileum and in human intestinal metaplasia.

How might it impact on clinical practice in the foreseeable future?

- This study provides a novel mechanism of CDX2 regulation, raising a new hypothesis for understanding the stability of this gastric preneoplastic lesion. Since the probability of intestinal metaplasia regression following eradication of $H$ pylori infection is low, in light of our results it may be necessary to interfere with the CDX2 autoregulatory loop, in addition to clearing the infection, in order to reverse intestinal metaplasia. This may have major implications when deciding on treatment for infected patients already harbouring this premalignant lesion. Identification of the self-sustainability of CDX2 is a major development in dealing with this and other cancer preneoplastic lesions that follow a transdifferentiation process as crucial steps during carcinogenesis.

animal models of $H$ pylori infection and subsequent lesions or induced gastric IM also show the progression from IM to gastric cancer. ${ }^{6-9}$ Understanding the mechanisms behind the establishment, maintenance and progression of IM is therefore of utmost importance. 
IM consists of the transdifferentiation of the gastric mucosa to an intestinal phenotype and depends on the expression of the homeobox transcription factor $\mathrm{CDX} 2$, the master gene for intestinal differentiation. ${ }^{10}{ }^{11}$ Under normal conditions CDX2 expression in adults is restricted to the intestine, but it becomes ectopically expressed in human IM lesions of the stomach, ${ }^{12} 13$ oesophagus ${ }^{14}{ }^{15}$ and gallbladder. ${ }^{16}$

Cdx2 homozygotic null mutant mice are not viable because embryos fail to implant, whereas $C d \times 2^{+/-}$mice develop noncancerous polyp-like lesions with focal loss of $\mathrm{Cdx} 2$ expression and development of gastric differentiation. ${ }^{17} 18$ Conversely, forced expression of $\mathrm{Cdx} 2$ in the stomach of transgenic mice leads to extensive IM, with subsequent progression to gastric cancer. ${ }^{9} 1920$ Further, CDX2 has been directly implicated in transcriptional regulation of intestinal terminal differentiation markers such as MUC2, ${ }^{21}$ LI-Cadherin ${ }^{22}$ and Sucrase-Isomaltase $^{23}$ among others. However, the molecular mechanisms regulating $\mathrm{CDX} 2$ expression in the establishment and maintenance of IM are not completely understood. The evidence so far suggests a complex regulation with involvement of multiple regulatory pathways. We recently demonstrated that key elements of the BMP pathway co-localised with CDX2 in IM and positively regulated CDX2 in gastric cell lines, ${ }^{24}$ and we showed a direct regulation of CDX2 expression by interaction of $H$ pylori with epithelial cells in an in vitro co-culture model. ${ }^{25}$ Both mechanisms fail to give any insight into the maintenance of CDX2 expression and the generally observed low reversibility of IM, even after eradication of $H$ pylori and clearance of the inflammatory response. ${ }^{26-28}$

$\mathrm{Xu}$ et al showed that CDX2 is able to transactivate its own promoter in vitro in a cell type-specific manner, ${ }^{29}$ suggesting a positive autoregulatory loop, but the importance of this process for CDX2 regulation in vivo in IM is yet to be established. Furthermore, the phenotype observed in the $C d \times 2^{+/-}$ mice clearly suggests a dose dependence on Cdx2 transcriptional activity compatible with a self-regulatory mechanism, since the germline loss of one allele with no structural second hit leads to total loss of expression of this gene focally. ${ }^{17}$ These results, together with the apparent stability of the CDX2-dependent IM phenotype, led us to hypothesise that CDX2 regulates its own expression through a positive autoregulatory loop in the gastric and intestinal contexts.

\section{METHODS}

\section{Cell culture, transient transfection and luciferase assays}

Human gastric carcinoma cell lines AGS (ATCC), MKN45 (JCRB0254, The RIKEN Cell Bank, Japan), GP202, ${ }^{30}$ IPA220, ${ }^{30}$ KatoIII (ATCC) and MKN28 (JCRB0253, The RIKEN Cell Bank, Japan) and intestinal carcinoma cell lines HCT116 (ATCC), HT29 (ATCC), HCT15 (ATCC) and CO115 ${ }^{31}$ were cultured under standard conditions. The reporter plasmids pCdx2-1Luc and pCdx2-9Luc containing $1 \mathrm{~kb}$ and $9.3 \mathrm{~kb}$ of the murine $\mathrm{Cdx} 2$ promoter, respectively, ${ }^{32}$ were used for cell transfection with Lipofectamine 2000 (Invitrogen, Carlsbad, USA) and luciferase assays. Cells were co-transfected with an expression vector for CDX2 or the corresponding empty vector, and a CMV- $\beta$-gal vector $(1 \mu \mathrm{g}$ DNA:1.5 $\mu$ l Lipofectamine). AGS cells were also cotransfected with the CDX2 expression vector or the corresponding empty vector, and the pCdx2-1luc wild-type, single and double mutated. Total extracts were prepared using $1 \times$ Reporter Lysis Buffer (Promega, Madison, USA) according to the manufacturer's instructions, and luciferase activity was measured in a 1450 Microbeta luminescence counter (Wallac, Perkin Elmer, Massachussets, USA). $\beta$-Galactosidase activity was measured to correct for transfection efficiency. Each experiment was carried out in triplicate at least twice and the results are expressed as mean $\pm \mathrm{SD}$ of representative triplicates.

AGS, MKN45, HCT116 and HeLa (ATCC) were transfected with a CDX2 expression vector containing two FLAG tags at the $\mathrm{N}$-terminus or the corresponding empty vector for RNA and protein analysis. MKN45 and HCT116 were also transfected with the CDX2 expression vector alone or together with the dominant negative truncated CDX2 form, miniCDX2,33 for RNA expression analysis. The amounts of DNA in each of these experimental conditions were equalised by adding the corresponding empty vector.

\section{Site-directed mutagenesis}

To introduce point mutations in the $\mathrm{pCdx} 2$-1luc gene promoter region, the QuickChange Site-directed mutagenesis protocol (Stratagene, Cedar Creek, USA) was used. The oligos used for this purpose are listed in table 1 . The double mutant was obtained by introducing the mutation MUT2 in the pCdx2-1luc MUT1. The PCR cycles were set as follows: $95^{\circ} \mathrm{C}$ for $30 \mathrm{~s} ; 16$ cycles of $95^{\circ} \mathrm{C}$ for $30 \mathrm{~s}, 55^{\circ} \mathrm{C}$ for $1 \mathrm{~min}, 68^{\circ} \mathrm{C}$ for $5 \mathrm{~min}$. Following PCR reaction, the products were incubated with $D p n I$ for $1 \mathrm{~h}$ at $37^{\circ} \mathrm{C}$ and transformed to $E$ coli competent cells. All the plasmids were checked by sequencing.

\section{RNA extraction and RT-PCR}

Total RNA was extracted using TRI Reagent (Sigma, St Louis, USA) and reverse transcription was performed with $5 \mu \mathrm{g}$ total RNA. PCR amplification was performed using the primer sets shown in table 1 and the products were separated in 1.5\% agarose gels. GAPDH levels were used as internal control. qPCR was performed using SYBR Green (endogenous CDX2) or Taqman (Applied Biosystems, Foster City, USA) reagents. TBP levels were used for normalisation and relative mRNA levels were calculated using the $\Delta \Delta \mathrm{Ct}$ method. The primers and probes used are listed in table 1. Each experiment was carried out in duplicate at least twice and the results are expressed as mean \pm SD.

\section{Protein extraction and western blot analysis}

Cells were lysated and the protein content was measured using standard methods. Protein extracts $(50 \mu \mathrm{g}$ for AGS and HCT116 and $70 \mu \mathrm{g}$ for MKN45) were analysed by standard SDS-PAGE, transferred to a nitrocellulose membrane (Amersham Biosciences, Buckinghamshire, UK) and blotted with primary antibodies (anti-CDX2, 1:500, Biogenex; anti-actin, 1:8000, SCBT) in $5 \%$ non-fat milk in $1 \times$ Tris buffered saline $0.02 \%$ Tween-20 (Sigma). Peroxidase-conjugated secondary antibodies (for CDX2 goat anti-mouse-HRP, 1:2000, and for actin goat anti-rabbitHRP, 1:2000, both from SCBT) were used and developed with the ECL detection kit (BioRad, Hercules, USA). Quantification of the western blots was performed using Bio-Rad Software Quantity One.

Chromatin immunoprecipitation in a gastric carcinoma cell line Chromatin immunoprecipitation (ChIP) was carried out using 90-95\% confluency IPA220 cells. Cells were washed with $1 \times$ phosphate buffered saline (PBS) and DNA/protein crosslinking was induced with $1 \%$ formaldehyde for $10 \mathrm{~min}$ at room temperature and terminated with glycine at $125 \mathrm{mM}$ final concentration. After being washed in $1 \times$ PBS containing protease inhibitors (Complete protease inhibitor cocktail; Sigma), the samples were sonicated in lysis buffer $(50 \mathrm{mM}$ Tris pH 8.0, 10 mM EDTA, 1\% SDS). The solubilised chromatin was incubated with anti-CDX2 antibody (BioGenex, The Hague, 
Table 1 Sequences of the oligonucleotides used for site-directed mutagenesis, RT-PCR, qPCR and chromatin immunoprecipitation (ChIP)

\begin{tabular}{|c|c|}
\hline Site-directed mutagenesis & $5^{\prime} \rightarrow 3^{\prime}$ \\
\hline Mutation $1^{*}$ & $\begin{array}{l}\text { S: CGATTGTTTAATGTAATAGTTTGGCAAAATGAATTGCTTTCC } \\
\text { AS: GGAAAGCAATTCATTTTGCCAAACTATTACATTAAACAATCG }\end{array}$ \\
\hline Mutation 2* & $\begin{array}{l}\text { S: TCTTGTAAACACTCGTTCCTCACGGAAGGCCGCCG } \\
\text { AS: CGGCGGCCTTCCGTGAGGAACGAGTGTTTACAAGA }\end{array}$ \\
\hline RT-PCR & $5^{\prime} \rightarrow 3^{\prime}$ \\
\hline Endogenous CDX2 & $\begin{array}{l}\text { F: CCAGGACGAAAGACAAATATCGA } \\
\text { R: GACTCTGCTAGACTCCTCAG }\end{array}$ \\
\hline Exogenous CDX2 & $\begin{array}{l}\text { F: CCAGGACGAAAGACAAATATCGA } \\
\text { R: CGACAAGCTTGACATTGAAGC }\end{array}$ \\
\hline GAPDH & $\begin{array}{l}\text { F: ACCATCTTCCAGGAGCGAG } \\
\text { R: GGATGACCTTGCCCACAG }\end{array}$ \\
\hline qPCR & $5^{\prime} \rightarrow 3^{\prime}$ \\
\hline Endogenous CDX2 & $\begin{array}{l}\text { F: GTGCTAAACCCCACCGTCAC } \\
\text { R: GACTCTGCTAGACTCCTCAG }\end{array}$ \\
\hline LI-Cadherin & TaqMan Gene Expression Assay CDH17 Hs00184865_m1 \\
\hline MUC2 & TaqManGene Expression Assay MUC2, Hs03005094_m1 \\
\hline TBP & TaqMan Gene Expression Assay TBP, Hs99999910_m1 \\
\hline human ChIP & $5^{\prime} \rightarrow 3^{\prime}$ \\
\hline pCDX2 region 1 & $\begin{array}{l}\text { F: GCATTAGCAGAAATTCTCTTTTC } \\
\text { R:GCATGTGGTAGAAGTTAGGCT }\end{array}$ \\
\hline pCDX2 region 2 & $\begin{array}{l}\text { F: CTAACTTCTACCACATGCCCA } \\
\text { R: GCATCTCTGACTTCATCTTACA }\end{array}$ \\
\hline pCDX2 region 3 & $\begin{array}{l}\text { F: GAGTTTCTTGACCGCCCTCTT } \\
\text { R: CCTCCAATCACAGGTTCAAAGA }\end{array}$ \\
\hline pCDX2 region 4 & $\begin{array}{l}\text { F: CTTTGAACCTGTGATTGGAGGT } \\
\text { R: CTCTACGCACAACCCCTCGAA }\end{array}$ \\
\hline pCDX2 region 5 & $\begin{array}{l}\text { F: CGAGGGGTTGTGCGTAGAGT } \\
\text { R: ACAGGCTGGCGTGCGGA }\end{array}$ \\
\hline pSucrase-Isomaltase & $\begin{array}{l}\text { F: GGCTGGTAAGGGTGCAATAA } \\
\text { R: GCCTGTTCTCTTTGCTATGTTG }\end{array}$ \\
\hline mouse ChIP & $5^{\prime} \rightarrow 3^{\prime}$ \\
\hline $\mathrm{pCdx} 2$ region 1 & $\begin{array}{l}\text { F: CAACGGTGGATTCATTCCG } \\
\text { R: GGAAGTATTTGTGCTGACACC }\end{array}$ \\
\hline $\mathrm{pCdx} 2$ region 2 & $\begin{array}{l}\text { F: GGTGTCAGCACAAATACTTCC } \\
\text { R: GTAATTAGTGGATGGCTGGG }\end{array}$ \\
\hline $\mathrm{pCdx2}$ region 3 & $\begin{array}{l}\text { F: CCCAGCCATCCACTAATTAC } \\
\text { R: GAAAAGACGATTCTACCTCCAG }\end{array}$ \\
\hline $\mathrm{pCdx} 2$ region 4 & $\begin{array}{l}\text { F: CGTTTCCAAACCCAGCTTCC } \\
\text { R: CCCCCAGAAACACGATTTGC }\end{array}$ \\
\hline $\mathrm{pCdx} 2$ region 5 & $\begin{array}{l}\text { F: GCAAATCGTGTTTCTGGGGG } \\
\text { R: GCGGCCTTACGTGATTAACG }\end{array}$ \\
\hline pSucrase-Isomaltase & $\begin{array}{l}\text { F: GATAGGCTTGTGAAAGTGCAAT } \\
\text { R: CCTGTAGTATCTGCTGTGTTG }\end{array}$ \\
\hline
\end{tabular}

The Netherlands) or control IgG overnight at $4^{\circ} \mathrm{C}$. Concomitantly, Protein G-Agarose beads (Roche, Indianapolis, USA) were blocked with $1 \%$ bovine serum albumin (BSA). Immunoprecipitated chromatin was then incubated with the beads for $2 \mathrm{~h}$ at $4^{\circ} \mathrm{C}$, washed with low salt buffer $(20 \mathrm{mM}$ Tris $\mathrm{pH} 8.0,150 \mathrm{mM}$ $\mathrm{NaCl}, 2 \mathrm{mM}$ EDTA, $0.1 \%$ SDS, 1\% Triton X-100), high salt buffer $(20 \mathrm{mM}$ Tris $\mathrm{pH}$ 8.0, $0.5 \mathrm{M} \mathrm{NaCl}, 2 \mathrm{mM}$ EDTA, 0.1\% SDS, $1 \%$ Triton X-100), LiCl buffer (10 mM Tris pH 8.0, 0.25 M LiCl, $1 \mathrm{mM}$ EDTA, 1\% NP-40, 1\% NaDOC) and TE buffer, and eluted with elution buffer ( $\left.0.1 \mathrm{M} \mathrm{Na}_{2} \mathrm{CO}_{3}, 1 \% \mathrm{SDS}\right)$. Reversion of the crosslink was performed overnight at $65^{\circ} \mathrm{C}$ and DNA was recovered using GFX DNA purification columns (GE Healthcare, Buckinghamshire, UK). The DNA was analysed by PCR using the primer pairs listed in table 1.

\section{Immunohistochemistry in frozen tissue sections}

Frozen sections were fixed in cold methanol and hydrated, followed by immunostaining as previously described. ${ }^{12}$ The primary antibody against CDX2 (BioGenex) was diluted 1:50 in $5 \%$ BSA.

\section{Chromatin immunoprecipitation in mouse ileum and in human intestinal metaplasia (IM)}

For mouse tissue ChIP, a wild-type mouse was killed and the intestine removed and washed in cold $\mathrm{NaCl} 9 \%$. The distal ileum mucosa was scraped using a glass slide and fixed in 1\% formaldehyde. The Magna ChIP Kit (Upstate Biotechnology, Millipore, Temecula, USA) was then used according to the manufacturer's guidelines. Immunoprecipitation was performed with $2 \mu \mathrm{g}$ mouse monoclonal anti-CDX2 antibody (Biogenex) and normal mouse IgGs (Invitrogen) were used as negative control. Extracted DNA was then analysed by qPCR with primers covering the mouse $\mathrm{Cdx} 2$ proximal promoter (table 1) and the values normalised against the IgG negative control.

IM tissue samples were collected from the stomach of three gastrectomised patients diagnosed with gastric adenocarcinoma who underwent surgery in Hospital S. João, Porto, Portugal, authorised by informed consent from the Tumour Bank of Hospital S. João, Porto, Portugal. The whole surgical specimens were briefly washed with water for removal of blood. For case 1 , four tissue samples were collected from the proximal and 
Figure 1 Regulation of a $1 \mathrm{~kb}$ and a $9.3 \mathrm{~kb}$ Cdx2 promoter by CDX2. Co-transfection experiments were performed in the presence of a $1 \mathrm{~kb}$ or $9.3 \mathrm{~kb} \mathrm{Cdx} 2$ promoter construct and a CDX2 expression vector in a panel of five gastric and four intestinal carcinoma cell lines. The values obtained were corrected for transfection efficiency with $\beta$-galactosidase activity and the luciferase activities obtained with transfection of the empty expression vector were referred to as 1 .

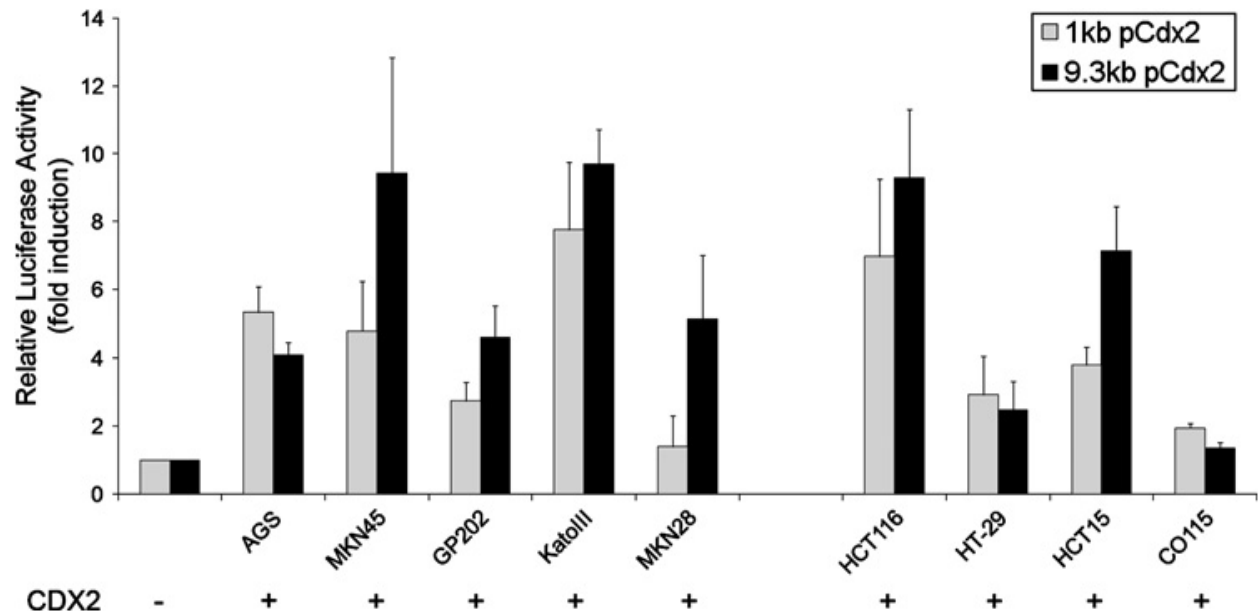

distal areas adjacent to the tumour and, for cases 2 and 3, two samples were collected from each. The mucosa was separated from the muscle layer, washed in cold $1 \times$ PBS with Complete protease inhibitor cocktail (Roche). The tissue segment was then cut into smaller pieces to facilitate fixation and fixed in $1 \%$ formaldehyde and fast frozen in liquid nitrogen. Without thawing, the tissue was pulverised using a Biopulverizer (Biospec Products, Bartlesville, USA) to facilitate posterior lysis. Following this step, the EZ Magna ChIP Kit (Upstate Biotechnology, Millipore) was used according to the manufacturer's recommendations. Immunoprecipitation was performed using the same antibody and control as above. Extracted DNA was then analysed by qPCR with primers covering the human CDX2 proximal promoter (table 1) and the values normalised against the IgG negative control. A second sample was collected adjacent to each of the previous samples, frozen in OCT and stained with haematoxylin and eosin to check for the presence of IM.

\section{RESULTS \\ CDX2 regulates its own promoter in gastric and intestinal carcinoma cell lines}

To determine whether CDX2 is able to activate transcriptionally its own promoter, the reporter plasmids pCdx2-1Luc and pCdx2-9Luc containing a $1 \mathrm{~kb}$ and a $9.3 \mathrm{~kb}$ promoter fragment of the murine Cdx2 gene were used for transfection and luciferase assays in a panel of gastric and intestinal carcinoma cell lines in combination with a CDX2 expression vector or the corresponding control empty vector. The cell lines used were AGS, MKN45, GP202, KatoIII and MKN28 (gastric) and HCT116, HT-29, HCT15 and CO115 (intestinal). CDX2 transactivates its own promoter at different levels, depending on the cellular context. Transcriptional inductions of the $9.3 \mathrm{~kb}$ promoter ranged from 1.4-fold in CO115 up to about 9-fold in MKN45, KatoIII and HCT116, and for the $1 \mathrm{~kb}$ promoter they ranged from 1.4-fold in MKN28 up to about 7-fold in KatoIII and HCT116 (figure 1). Using a series of deletion mutants
Figure 2 Active CDX2 cis-elements on the CDX2 promoter. (A) Chromatin immunoprecipitation was performed with an isotype control immunoglobulin $\mathrm{G}(\mathrm{IgG})$ and with an anti-CDX2 antibody. PCR amplification of five regions within the proximal CDX2 promoter was performed to detect CDX2-bound DNA. A fraction of the chromatin preparation $(1 \%)$ was used as input in the reaction. (B) Site-directed mutagenesis was used to introduce mutations 1 and 2, alone or together, in a construct of $1 \mathrm{~kb}$ of the $\mathrm{mCdx} 2$ proximal promoter.

Co-transfection experiments were then performed in the presence of the $1 \mathrm{~kb}$ promoter construct, wild-type, single or double mutated, and a CDX2 expression vector in AGS cells. The values obtained were corrected for transfection efficiency with $\beta$-galactosidase activity and the luciferase activities obtained with transfection of the empty expression vector were referred to as 1 .
A
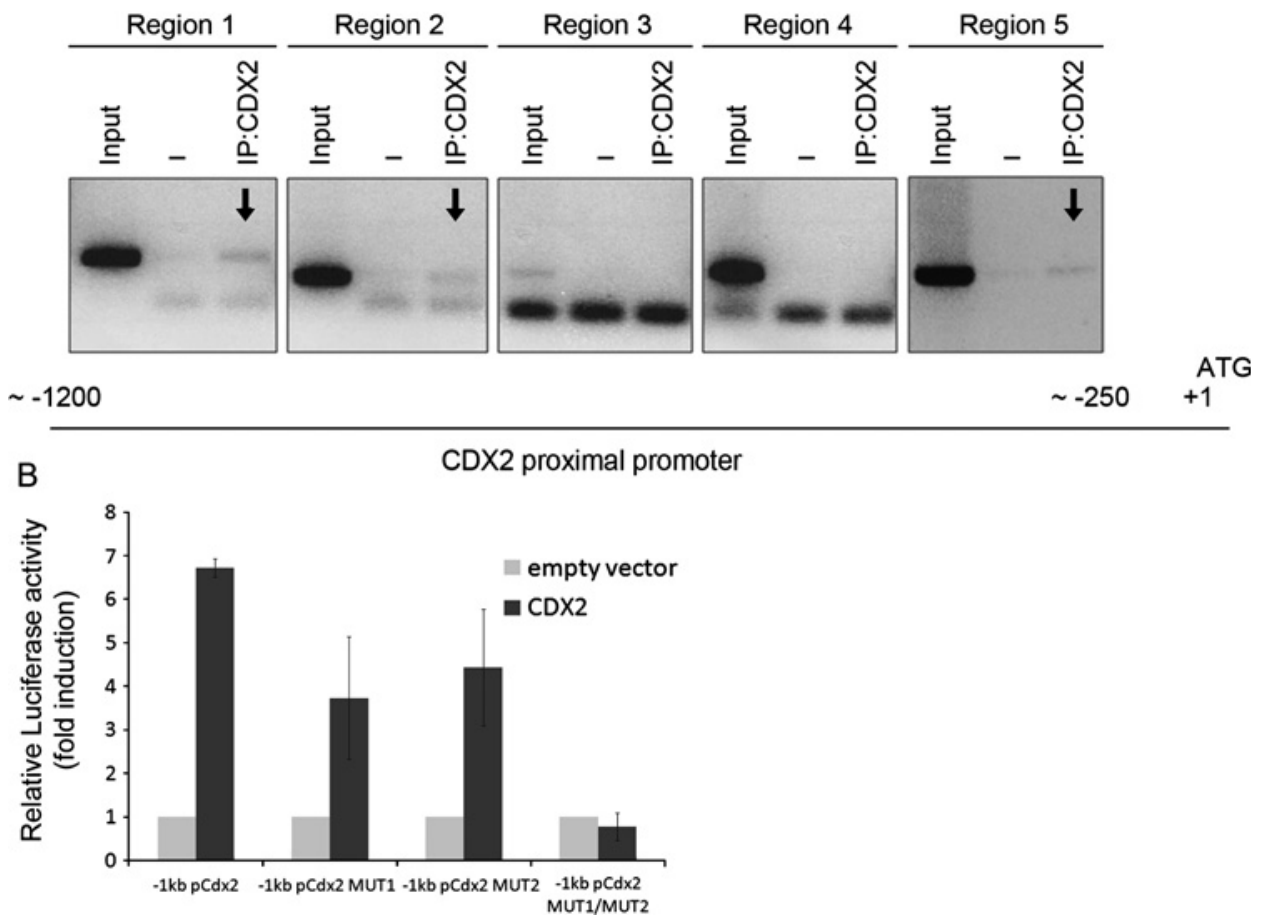
comprising $2 \mathrm{~kb}$ up to $8 \mathrm{~kb}$ of the $\mathrm{Cdx} 2$ promoter, we found CDX2 transactivation of its own promoter in all the constructs. There was no obvious pattern of increasing transactivation along increasing promoter lengths that could contribute to mapping potential CDX2 binding sites. The levels of transactivation varied among cell lines independently of basal CDX2 expression, as already found for the $1 \mathrm{~kb}$ and $9.3 \mathrm{~kb}$ promoters (data not shown).

\section{Identification of active CDX2 cis-elements within the CDX2 promoter}

In order to demonstrate that the CDX2 transcription factor binds directly to its own promoter, ChIP was performed in IPA220 cells, which is the cell line with the highest CDX2 expression. $^{24}$ Since CDX2 was able to transactivate its own promoter in the $1 \mathrm{~kb}$ construct, we studied the most proximal region of the CDX2 promoter and found several putative binding sequences for CDX2, more or less conserved relative to the consensus CDX2 binding sequence. ${ }^{34}$ Five regions within the first 1200 nucleotides were evaluated in the precipitated DNA. The results show that CDX2 protein is present in three of the regions-the most proximal and the two most distal ones (figure 2A). To examine the functional role of these CDX2 binding sites, we chose two sequences conserved between mouse and human to mutate by site-directed mutagenesis. These two sites (-954/-952 and -293/-292) were mutated independently and together in the $-1 \mathrm{~kb}$ mouse $\mathrm{Cdx} 2$ promoter construct. The wild-type and the three mutated Cdx2 promoter constructs were co-transfected with CDX2 in AGS cells and promoter activity was evaluated by a luciferase assay. The results show that each independent mutation of the putative CDX2 binding
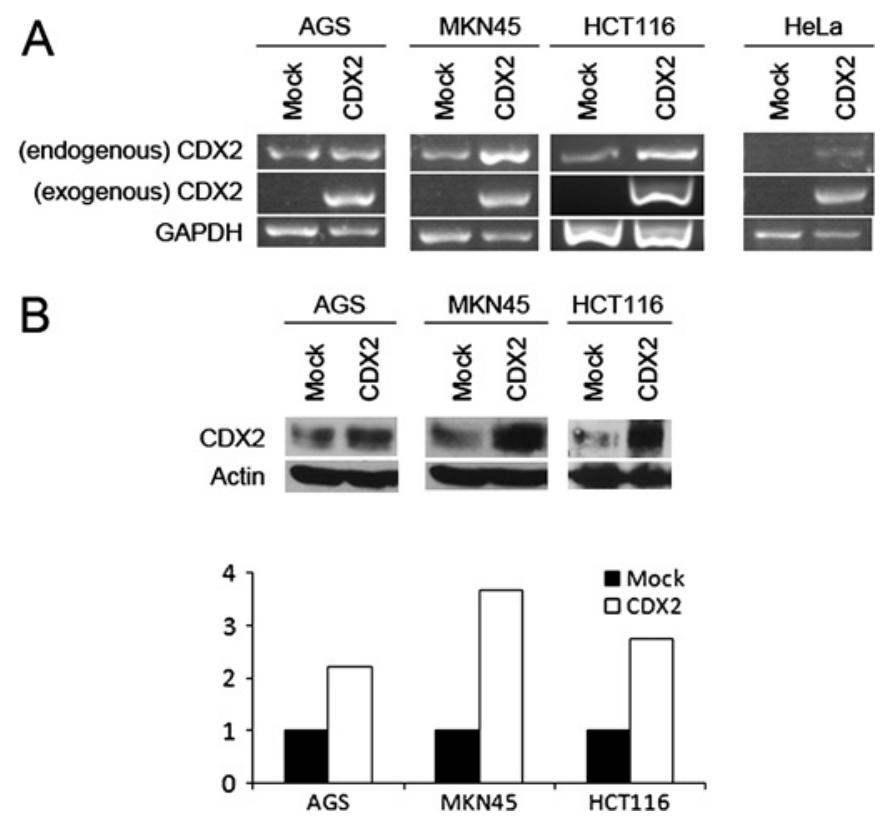

Figure 3 Effect of CDX2 transfection on endogenous CDX2 levels in gastrointestinal and non-gastrointestinal cell lines. (A) Endogenous and exogenous CDX2 mRNA level in AGS, MKN45, HCT116 and HeLa cell lines upon transfection with either an empty vector or a vector expressing CDX2. GAPDH was used as mRNA level control. (B) Western blots showing endogenous levels of CDX2 in AGS, MKN45 and HCT116 cell lines upon transfection with either an empty vector or a vector expressing CDX2. $\beta$-actin was used as loading control. The lower part of (B) shows quantification of the western blots. sites located within the positive binding regions identified by ChIP leads to decreased promoter activity of about $40 \%$, whereas the double mutant leads to complete abrogation of promoter activation (figure $2 \mathrm{~B}$ ).

\section{Transfection of CDX2 increases the endogenous level of CDX2 in a panel of cell lines}

In order to study the ability of CDX2 to regulate its own expression in the endogenous context of cell lines, the gastric AGS and MKN45 and the intestinal HCT116 cell lines were transfected with CDX2 expression vector or the corresponding empty vector and endogenous CDX2 levels were evaluated at the mRNA and protein levels. The endogenous and vector mRNA gene products are distinguished using specific primer pairs, and corresponding proteins are discriminated by molecular weight which differs due to the two FLAG tags encoded by the expression vector. The results show that there was a clear increase in endogenous CDX2 mRNA expression on CDX2 transfection in MKN45 and HCT116, with a smaller effect in AGS (figure $3 \mathrm{~A}$ ). The same results were observed at the protein level where endogenous CDX2 increased by 2.2, 2.7 and 3.7 times in AGS, HCT116 and MKN45 cells, respectively (figure $3 \mathrm{~B}$ ). To determine whether this effect was cell contextspecific, the same transfection experiment was performed in the non-gastrointestinal HeLa cell line which does not express CDX2 endogenously. RT-PCR results show that CDX2 transfection activates the endogenous expression of CDX2 in these cells (figure $3 \mathrm{~A}$ ).

\section{A dominant-negative form of CDX2 abrogates its own regulation}

To confirm the positive effect of CDX2 in regulating its own endogenous expression, we set up an experimental context using a dominant negative form of CDX2. ${ }^{33}$ This is a truncated form of the CDX2 protein (miniCDX2) which competes for the DNA binding with its wild-type counterpart but lacks the transactivation activity. When transfecting miniCDX2 together with the wild-type form in two cell lines (MKN45 and HCT116), we found almost complete abrogation of the inducing activity of CDX2 on its targets MUC2 (figure 4A) and LICadherin (figure 4B), thus confirming its dominant negative activity, and on endogenous CDX2 (figure 4C) in accordance with the previous results.

\section{Cdx2 is bound to its own promoter in the mouse ileum and in human intestinal metaplasia}

In order to confirm the relevance of the autoregulatory mechanism in vivo, ChIP was performed against Cdx2 in the mouse distal ileum, which is one of the intestinal segments with highest $\mathrm{Cdx} 2$ expression. ${ }^{32} \mathrm{~A}$ series of primer pairs covering about 1200 nucleotides of the $\mathrm{Cdx} 2$ proximal promoter region was designed, together with one encompassing the Cdx2 binding site on the Sucrase-Isomaltase promoter ${ }^{32}$ as a positive control. The results show that $\mathrm{Cdx} 2$ is bound to the promoter region detected with the primer pair 5 (figure $5 \mathrm{~A}$ ), which covers a region corresponding to the more distal positive region detected by ChIP in the human cell line. As expected, Cdx2 was also bound to Sucrase-Isomaltase promoter.

We further aimed to confirm the relevance of this regulatory pathway in human IM by performing a ChIP experiment in samples of IM. Since CDX2 is exclusively present in the stomach in foci of IM, ChIP was performed using the whole mucosa fragment containing both normal and metaplastic glands. As shown in figure $5 \mathrm{~B}$ for case 1 , we collected four mucosa samples 


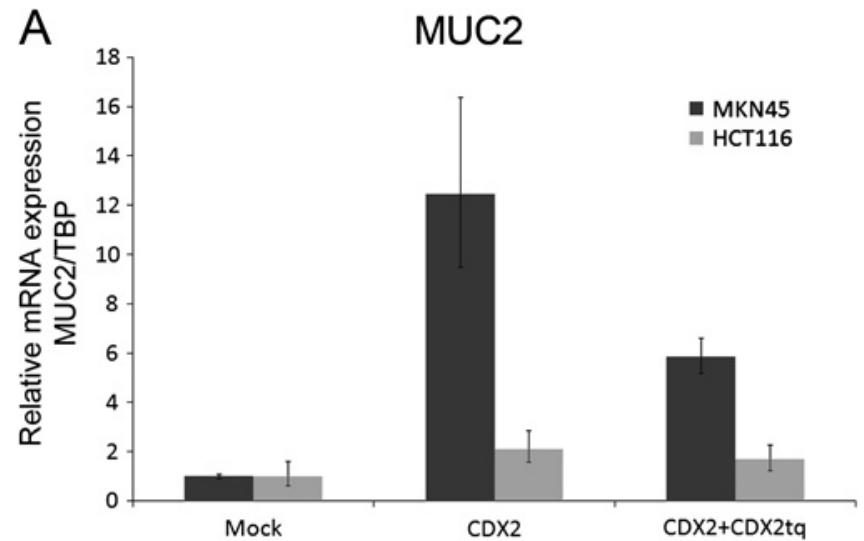

B LI-Cadherin
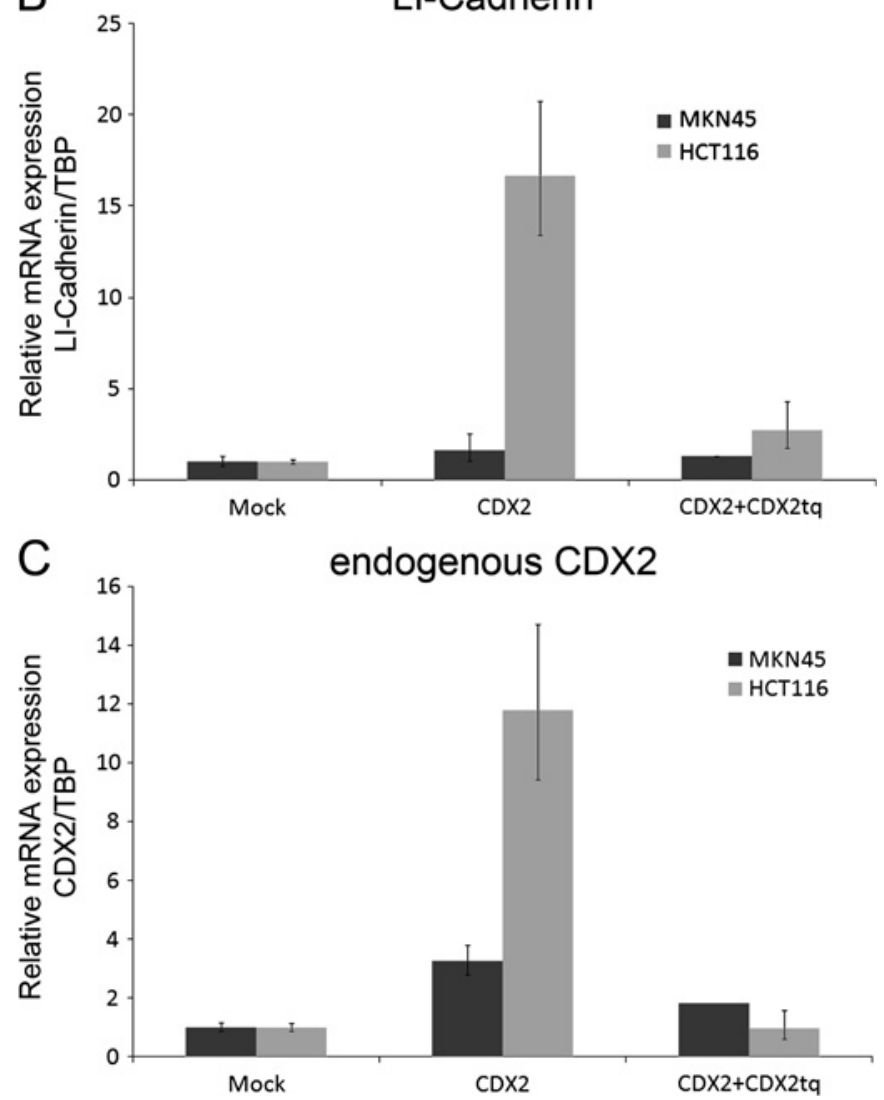

Figure 4 Effect of a dominant negative form of CDX2 on CDX2 targets and its endogenous expression. Fold increase of (A) MUC2,

(B) LI-Cadherin and (C) endogeneous CDX2 mRNA in MKN45 and HCT116 cells transfected with CDX2 alone or together with a dominant negative form, evaluated by qPCR. The values obtained with mocktransfected cells were referred to as 1. mRNA levels were normalised to the corresponding TBP mRNA level.

(numbered 1-4) from different areas surrounding the tumour. After observation of samples immediately adjacent to them, samples 1 and 2 were excluded because they did not have clear foci of IM and ChIP was performed on samples 3 and 4, both of which contained several foci of IM (figure 5C,D). We confirmed, as previously described, ${ }^{12}$ that CDX2 protein is only present in areas of IM (figure $5 \mathrm{C} 3, \mathrm{D} 3$ ). The results obtained from the immunoprecipitated DNA in sample 3 show that CDX2 is bound to its promoter in two regions, one more proximal and one further upstream, separated by a region with no binding (figure $5 \mathrm{E}$ ). The regions that were positive in IM correspond to those found in the human cell line IPA220. The most distal regions found in human cells correspond to the region found positive in the mouse ileum. Furthermore, CDX2 is bound to the human Sucrase-Isomaltase promoter which may also be used as a positive control in IM (figure $5 \mathrm{E}$ ). The results for sample 4 were similar, although promoter region 3 , which was negative in sample 3 , showed positive binding but at a much lower level than in the other regions (figure $5 \mathrm{~F}$ ).

The results for cases 2 and 3 were in line with those for case 1 (see online supplement), showing an enrichment in immunoprecipitated DNA in IM samples and, as expected, negative results for the normal gastric mucosa which contains no CDX2.

\section{DISCUSSION}

In the present study we show that CDX2 is autoregulated in vivo and behaves as its own transcriptional target in the gastric and intestinal context. Our results confirm and extend the previously shown CDX2 transactivation of its own promoter in a pancreatic and an intestinal cell line. ${ }^{29}{ }^{35}$ In this study, CDX2 was shown to transactivate a $9.3 \mathrm{~kb} \mathrm{Cdx} 2$ promoter which better mimics the endogenous promoter by covering a much more extensive regulatory region in a panel of gastrointestinal cell lines. Nevertheless, we were able to demonstrate transactivation in the same panel of cell lines from the much shorter $1 \mathrm{~kb} \mathrm{Cdx} 2$ promoter, indicating that the activity of CDX2 on its own promoter occurs in the most proximal sequences. The levels of transactivation varied among cell lines, so we cannot exclude the possibility that context-specific co-factors-either activators or repressors-may intervene in the autoregulatory process. CDX1, which is also autoregulated, ${ }^{36}$ was shown to depend on direct interaction with LEF1 and subsequent complex binding to LEF/TCF response elements on the CDX1 promoter for autoregulation to occur. ${ }^{37}$

CDX2 usually binds AT-rich sequences, more or less conserved with the consensus TTTAT, which are often in close proximity to the TATA box in many CDX2 target genes. We used this criterion to define the putative CDX2 binding sites within the regions showing CDX2 binding as assessed by ChIP, which were selected for posterior mutational analysis. Our results show that the two selected sites had some degree of redundancy in CDX2 autoregulation since mutation of each of them alone led to a reduction in CDX2 transactivation of its own promoter of only about $40 \%$. However, when mutating both sites together, we were able to abrogate the positive activity of CDX2 on its own promoter, thus showing that CDX2 binds to its promoter and also directly transactivates it in vitro.

We further studied this autoregulation by assessing CDX2 activity on its endogenous levels. In addition to increasing its own endogenous expression in gastric and intestinal cell lines, CDX2 also initiated its own mRNA expression in the nonCDX2-expressing cervical HeLa cell line. Analysing the results obtained for the gastrointestinal cell lines, it is worth noting that the lower the starting endogenous CDX2 level, the greater is the effect. This is in agreement with previous reports ${ }^{38}$ that cell lines cannot cope with too high an expression of CDX2, possibly owing to induction of proliferation arrest and apoptosis. ${ }^{11}$ On the other hand, the results with the HeLa cell line show that CDX2 is able to initiate its own expression in a non-gastrointestinal setting and without further stimulus, which suggests that the autoregulatory mechanism is sufficient to maintain its expression. The results obtained with miniCDX2 reinforce this observation, showing that CDX2 behaves like its other targets. 


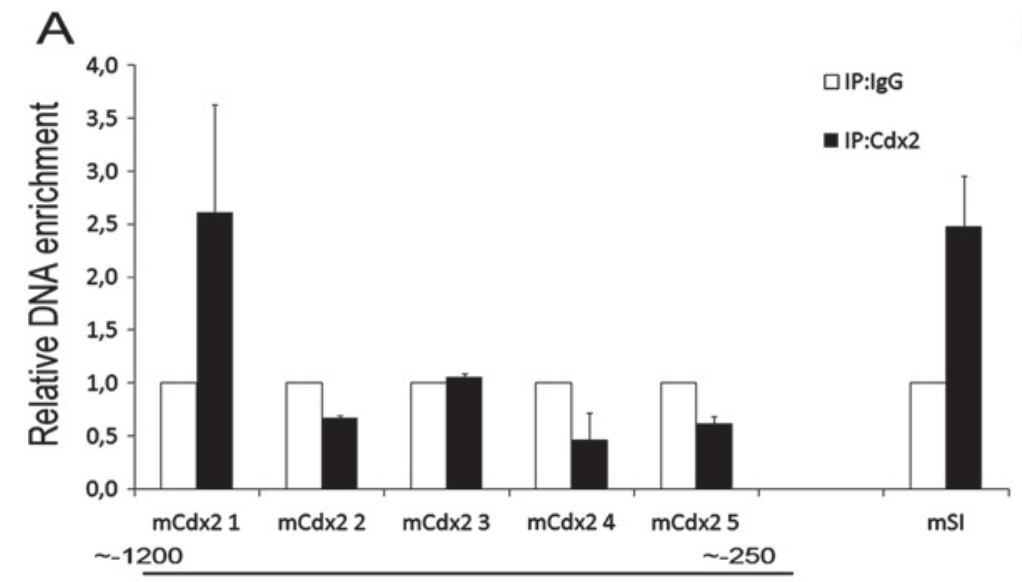

B

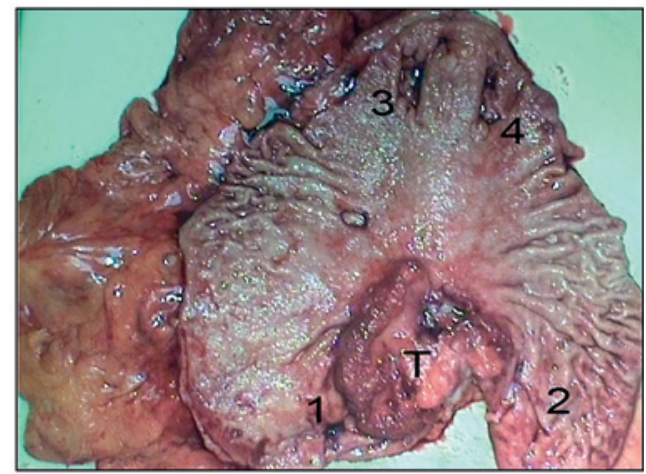

$\mathrm{mCd} 2$ proximal promoter
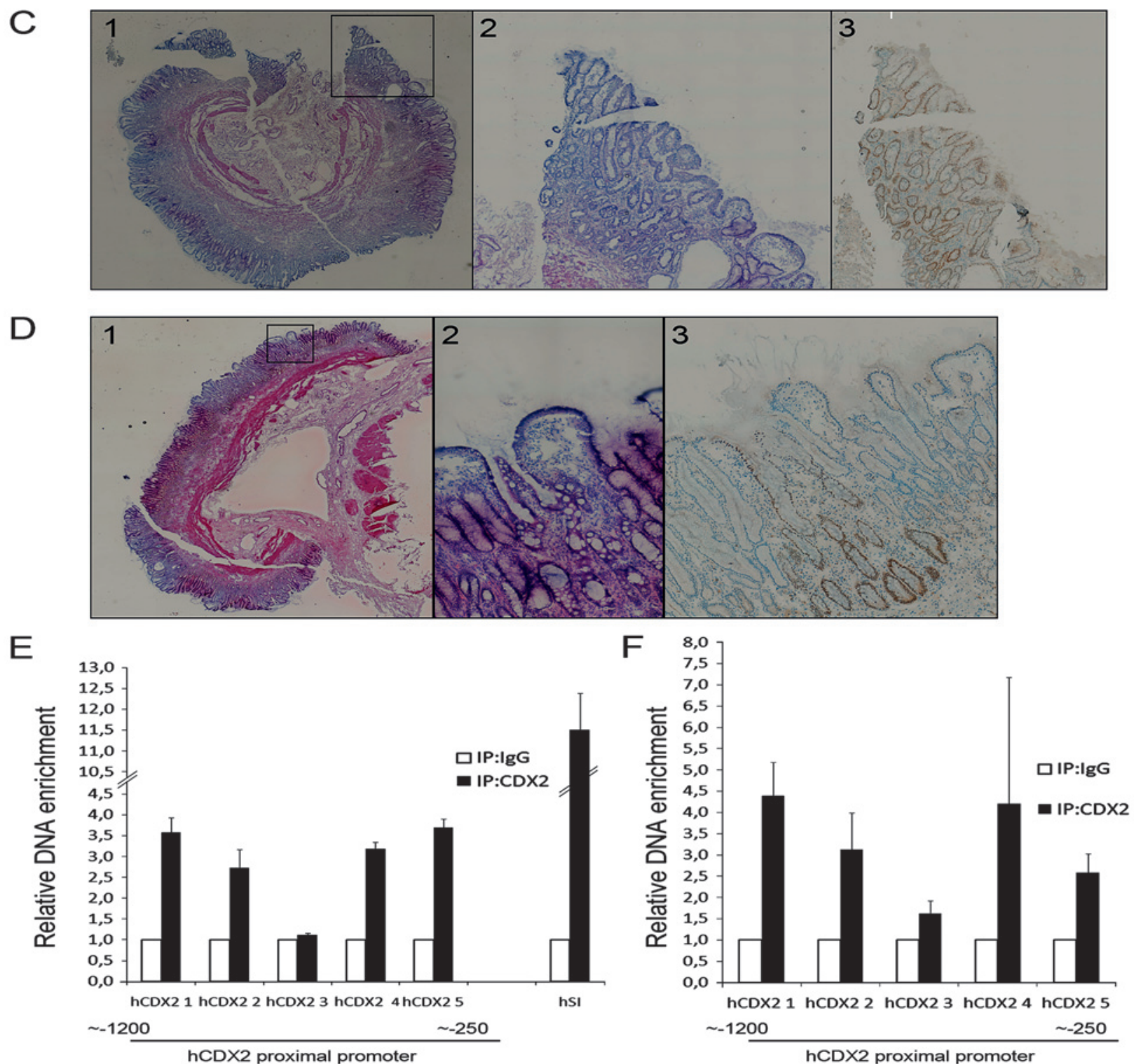

Figure 5 CDX2 binding to its own promoter in mouse ileum and human intestinal metaplasia. Chromatin immunoprecipitation was carried out with an isotype control immunoglobulin $\mathrm{G}(\mathrm{IgG})$ and with an anti-CDX2 antibody in DNA from (A) mouse ileum and two samples of human intestinal metaplasia $(E$ and $F)$. Purified DNA was analysed by qPCR using specific primers covering five regions of the proximal mouse and human CDX2 promoters. Fold enrichments are expressed as ratios of the IP:CDX2 signal to that of the IP:IgG signal and calculated by extrapolation from a standard curve of input DNA dilutions. (B) Whole gastrectomised stomach showing the samples taken for analysis $(1-4)$ and the adenocarcinoma (T). (C) H\&E stain and (D) immunohistochemical detection of CDX2 in fragments 3 and 4, respectively: (C1,D1) H\&E stain of the whole fragments (magnification $\times 4)$; (C2, D2) foci of intestinal metaplasia (magnification $\times 20)$; $(C 3, D 3)$ CDX2 immunostaining in the same areas. 
Furthermore, we found that $\mathrm{Cdx} 2$ binds its own promoter in the mouse intestine and in human IM, which suggests that autoregulation of CDX2 occurs in vivo and may have a role in the maintenance of this lesion.

The first suggestion that CDX2 autoregulation could be relevant in vivo came from heterozygous $C d \times 2$ null mice. ${ }^{18}$ While absent in the intestinal polyps, $\mathrm{Cdx} 2$ expression from one single allele is retained in the surrounding normal intestinal epithelium. These observations suggest that the combination of a single functional allele lacking some external signal/co-factor might result in failure to reach a certain threshold of expression leading to complete loss of $\mathrm{Cdx} 2$ and polyp development, which is in line with a self-regulatory mechanism. Moreover, the extent, time and location of the defects varied among animals, suggesting that a critical threshold may have to be reached for autoregulation to take place. In this study we show that Cdx2 is autoregulated in the intestine, which is in line with these observations.

It has been well demonstrated over the years that CDX2 plays a crucial role in the determination of the intestinal phenotype during embryogenesis and its maintenance postnatally. Moreover, focal gains and losses of CDX2 expression induce a shift in phenotype from gastric/oesophagus to intestinal and vice versa. One well documented example is IM of the stomach which is triggered by CDX2. Gastric IM is a preneoplastic lesion associated with a higher risk of gastric cancer development. ${ }^{3-5}$ Despite ongoing controversy, once present, gastric IM rarely seems to regress. ${ }^{26-2839}$ This remains true even after conditions favouring its appearance-such as $H$ pylori infection and the concomitant inflammation-have been treated, and has been shown in studies by Asfeldt et $a l^{26}$ and by Wong et $a l^{27}$ in addition to ours. It is, in fact, impossible to follow the fate of a particular IM focus after $H$ pylori eradication and inflammation clearance, but the abovementioned studies show that IM persists in most individuals after eradication therapy. The present study provides a putative explanation for this, based on the maintenance of CDX2 expression through an autoregulatory loop which is independent of the initial trigger, thus maintaining the intestinal phenotype. It is tempting to speculate that, in addition to clearing the infection and inflammation, it is probably necessary to interfere with the CDX2 autoregulatory loop in order to reverse IM.

In the apparently rare setting of loss of CDX2 by the metaplastic glands and their disappearance from the gastric mucosa, further studies will be needed to show if the cells transdifferentiate back to the gastric phenotype or rather are overgrown by the surrounding normal gastric glands. ${ }^{40}$ From the mechanistic and developmental point of view, it would be interesting to discriminate fully between these two processes. However, from the clinical point of view, the major interest is to be able to interfere with the maintenance of IM and therefore to interrupt the gastric carcinogenic pathway.

Together, the results presented in this study clearly show that CDX2 regulates its own expression in the gastrointestinal context and is bound to its own promoter in the mouse intestine and in human IM. This regulatory mechanism may have an impact on the stability of human IM and, possibly, on the inevitable progression of the gastric carcinogenesis pathway.

Acknowledgements The authors are grateful to Professor Fátima Carneiro, Head of the Pathology Department at Hospital S. João, Porto, Portugal and to Dr Helena Coutinho and Dr Elisabete Rios for assistance in human sample collection.

Funding This work was supported by Fundação para a Ciência e a Tecnologia (FCT) (Project PTDC/SAU-OBD/64490/2006) financed by Programa Operacional Ciência e
Inovação 2010 do Quadro Comunitário de Apoio III and FEDER. IPATIMUP is an Associate Laboratory of the Portuguese Ministry of Science, Technology and Higher Education and is partially supported by FCT. RB was supported by FCT (SFRH/BD/29622/2006).

Competing interests None.

Ethics approval This study was conducted with the approval of the Hospital S. João, Porto, Portugal.

Provenance and peer review Not commissioned; externally peer reviewed.

\section{REFERENCES}

1. Parkin DM. The global health burden of infection-associated cancers in the year 2002. Int J Cancer 2006:118:3030-44.

2. Correa P. Human gastric carcinogenesis: a multistep and multifactorial process-First American Cancer Society Award Lecture on Cancer Epidemiology and Prevention. Cancer Res 1992;52:6735-40.

3. Uemura N, Okamoto $\mathrm{S}$, Yamamoto $\mathrm{S}$, et al. Helicobacter pylori infection and the development of gastric cancer. N Engl J Med 2001:345:784-9.

4. Guarner J, Mohar A, Parsonnet J, et al. The association of Helicobacter pylori with gastric cancer and preneoplastic gastric lesions in Chiapas, Mexico. Cancer 1993; 71:297-301.

5. de Vries AC, van Grieken NC, Looman CW, et al. Gastric cancer risk in patients with premalignant gastric lesions: a nationwide cohort study in the Netherlands. Gastroenterology 2008;134:945-52.

6. Honda S, Fujioka T, Tokieda M, et al. Development of Helicobacter pylori-induced gastric carcinoma in Mongolian gerbils. Cancer Res 1998;58:4255-9.

7. Watanabe T, Tada M, Nagai H, et al. Helicobacter pylori infection induces gastric cancer in mongolian gerbils. Gastroenterology 1998;115:642-8.

8. Zheng 0, Chen XY, Shi Y, et al. Development of gastric adenocarcinoma in Mongolian gerbils after long-term infection with Helicobacter pylori. J Gastroentero Hepatol 2004;19:1192-8.

9. Mutoh H, Sakurai S, Satoh K, et al. Development of gastric carcinoma from intestinal metaplasia in Cdx2-transgenic mice. Cancer Res 2004;64:7740-7.

10. Silberg DG, Swain GP, Suh ER, et al. Cdx1 and cdx2 expression during intestinal development. Gastroenterology 2000;119:961-71.

11. Suh E, Traber PG. An intestine-specific homeobox gene regulates proliferation and differentiation. Mol Cell Biol 1996;16:619-25.

12. Almeida R, Silva E, Santos-Silva F, et al. Expression of intestine-specific transcription factors, CDX1 and CDX2, in intestinal metaplasia and gastric carcinomas. J Pathol 2003:199:36-40.

13. Bai Y0, Yamamoto $\mathrm{H}$, Akiyama $\mathrm{Y}$, et al. Ectopic expression of homeodomain protein CDX2 in intestinal metaplasia and carcinomas of the stomach. Cancer Lett 2002:176:47-55.

14. Eda A, Osawa H, Satoh K, et al. Aberrant expression of CDX2 in Barrett's epithelium and inflammatory esophageal mucosa. J Gastroenterol 2003;38:14-22.

15. Phillips RW, Frierson HF Jr, Moskaluk CA. Cdx2 as a marker of epithelial intestinal differentiation in the esophagus. Am J Surg Pathol 2003;27:1442-7.

16. Osawa H, Kita H, Satoh K, et al. Aberrant expression of CDX2 in the metaplastic epithelium and inflammatory mucosa of the gallbladder. Am J Surg Pathol 2004;28:1253-4

17. Beck F, Chawengsaksophak K, Waring $P$, et al. Reprogramming of intestinal differentiation and intercalary regeneration in $\mathrm{Cdx} 2$ mutant mice. Proc Natl Acad Sci USA 1999;96:7318-23.

18. Chawengsaksophak K, James R, Hammond VE, et al. Homeosis and intestinal tumours in Cdx2 mutant mice. Nature 1997;386:84-7.

19. Mutoh H, Hakamata Y, Sato K, et al. Conversion of gastric mucosa to intestinal metaplasia in Cdx2-expressing transgenic mice. Biochem Biophys Res Commun 2002;294:470-9

20. Silberg DG, Sullivan J, Kang E, et al. Cdx2 ectopic expression induces gastric intestinal metaplasia in transgenic mice. Gastroenterology 2002;122:689-96.

21. Mesquita $\mathbf{P}$, Jonckheere N, Almeida R, et al. Human MUC2 mucin gene is transcriptionally regulated by $\mathrm{Cdx}$ homeodomain proteins in gastrointestinal carcinoma cell lines. J Biol Chem 2003;278:51549-56.

22. Hinoi T, Lucas PC, Kuick R, et al. CDX2 regulates liver intestine-cadherin expression in normal and malignant colon epithelium and intestinal metaplasia. Gastroenterology 2002; 123:1565-77

23. Suh E, Chen L, Taylor J, et al. A homeodomain protein related to caudal regulates intestine-specific gene transcription. Mol Cell Biol 1994;14:7340-51.

24. Barros R, Pereira B, Duluc I, et al. Key elements of the BMP/SMAD pathway co-localize with CDX2 in intestinal metaplasia and regulate CDX2 expression in human gastric cell lines. J Pathol 2008;215:411-20

25. Barros R, Marcos N, Reis CA, et al. CDX2 expression is induced by Helicobacter pylori in AGS cells. Scand J Gastroenterol 2009;44:124-5.

26. Asfeldt AM, Steigen SE, Lochen ML, et al. The natural course of Helicobacter pylori infection on endoscopic findings in a population during 17 years of follow-up: the Sorreisa Gastrointestinal Disorder Study. Eur J Epidemiol 2009;24:649-58.

27. Wong BC, Lam SK, Wong WM, et al. Helicobacter pylori eradication to prevent gastric cancer in a high-risk region of China: a randomized controlled trial. JAMA 2004;291:187-94. 
28. Barros R, Peleteiro B, Almeida R, et al. Relevance of high virulence Helicobacter pylori strains and futility of CDX2 expression for predicting intestinal metaplasia after eradication of infection. Scand J Gastroenterol 2010;45:828-34.

29. $\mathbf{X u ~} \mathbf{F}$, Li H, Jin T. Cell type-specific autoregulation of the Caudal-related homeobox gene Cdx-2/3. J Biol Chem 1999;274:34310-16.

30. Gartner F, David L, Seruca R, et al. Establishment and characterization of two cell lines derived from human diffuse gastric carcinomas xenografted in nude mice. Virchows Arch 1996;428:91-8.

31. Carrel S, Sordat B, Merenda C. Establishment of a cell line (Co-115) from a human colon carcinoma transplanted into nude mice. Cancer Res 1976;36:3978-84.

32. Benahmed F, Gross I, Gaunt SJ, et al. Multiple regulatory regions control the complex expression pattern of the mouse Cdx2 homeobox gene. Gastroenterology 2008;135:1238-47. e1-3

33. Modica S, Morgano A, Salvatore L, et al. Expression and localisation of insulin receptor substrate 2 in normal intestine and colorectal tumours. Regulation by intestine-specific transcription factor CDX2. Gut 2009;58:1250-9.
34. Margalit Y, Yarus S, Shapira E, et al. Isolation and characterization of target sequences of the chicken CdxA homeobox gene. Nucleic Acids Res 1993;21:4915-22.

35. da Costa LT, He TC, Yu J, et al. CDX2 is mutated in a colorectal cancer with normal APC/beta-catenin signaling. Oncogene 1999;18:5010-14.

36. Prinos $\mathbf{P}$, Joseph $\mathrm{S}$, Oh K, et al. Multiple pathways governing Cdx1 expression during murine development. Dev Biol 2001;239:257-69.

37. Beland $\mathbf{M}$, Pilon $\mathrm{N}$, Houle $\mathbf{M}$, et al. Cdx1 autoregulation is governed by a novel Cdx1-LEF1 transcription complex. Mol Cell Biol 2004;24:5028-38.

38. Kong J, Nakagawa $\mathrm{H}$, Isariyawongse BK, et al. Induction of intestinalization in human esophageal keratinocytes is a multistep process. Carcinogenesis 2009;30:122-30.

39. Correa P, Fontham ET, Bravo JC, et al. Chemoprevention of gastric dysplasia: randomized trial of antioxidant supplements and anti-helicobacter pylori therapy. J Natl Cancer Inst 2000;92:1881-8.

40. Slack JM. Metaplasia and transdifferentiation: from pure biology to the clinic. Nat Rev Mol Cell Biol 2007;8:369-78.

\section{Editor's quiz: GI snapshot}

\section{The issue is tissue}

A 69-year-old man, otherwise well, except for ischaemic heart disease and asthma, presented with a 2 year history of crampy abdominal pain relieved with vomiting, intermittent diarrhoea up to 15 times per day and associated $20 \mathrm{~kg}$ weight loss. Physical examination revealed a soft abdomen with mild distension. Initial laboratory data were normal. Multiple stool cultures were negative. Plain abdominal radiography and CT revealed dilatation of multiple loops of small and large bowel.

Metabolic, autoimmune, infectious including HIV and tuberculosis), as well as a carcinoid screen including an octreotide scan were negative. Endoscopy was normal with no evidence of villous atrophy on duodenal biopsy. Colonoscopy and biopsies were normal. Double balloon enteroscopy revealed an area of lymphangiectasia with jejunal biopsies revealing non-specific mild jejunitis. There was no evidence of malignancy on serial imaging including positron emission tomography scan.

Repeated presentations led finally to laparotomy where there was no evidence of mechanical obstruction. At laparotomy,

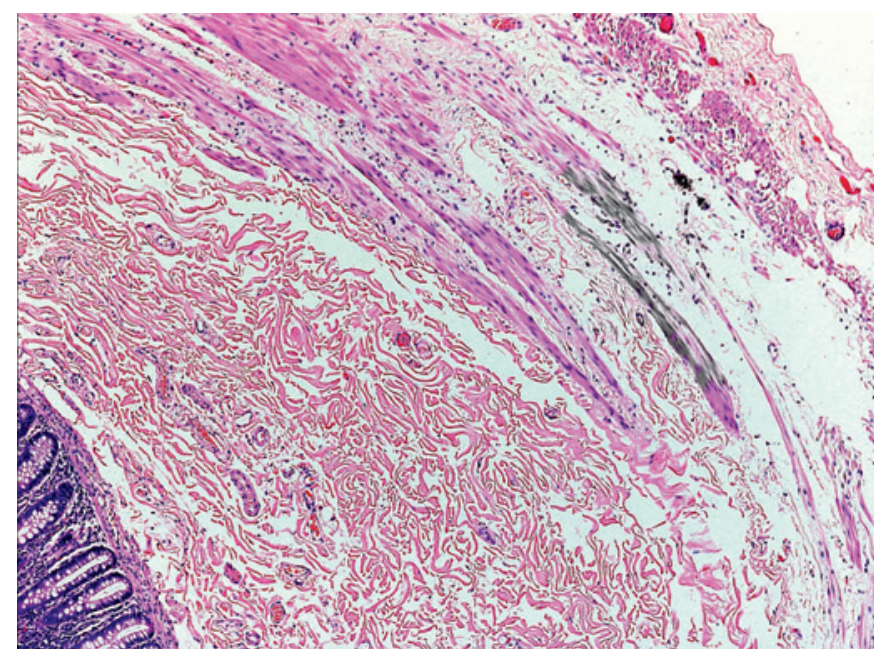

Figure 1 Full thickness biopsy of small bowel $(5 \times$ magnification).

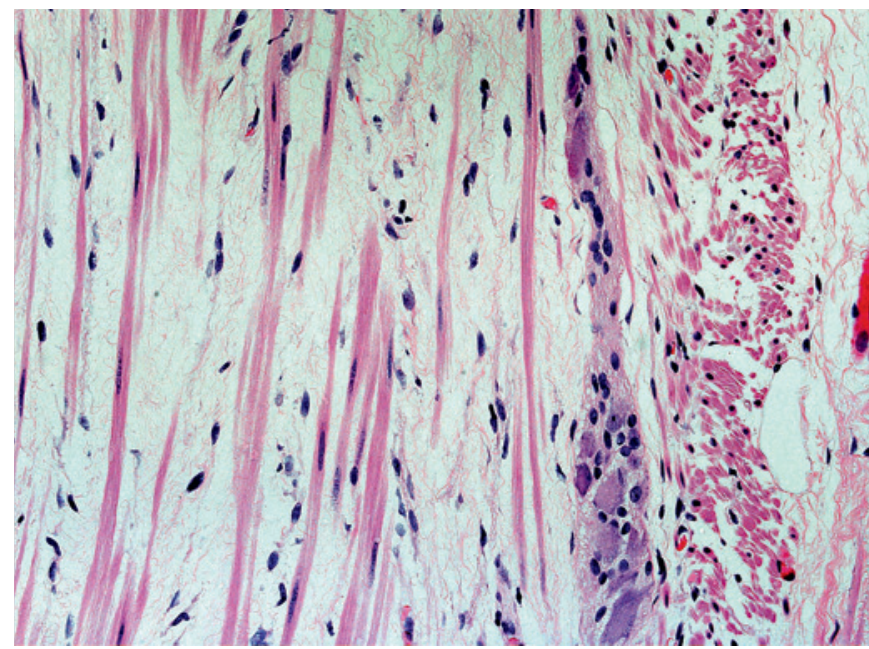

Figure 2 Full thickness biopsy of large bowel (20× magnification).

full-thickness biopsies of small and large bowel (figures 1 and 2) were obtained.

\section{QUESTION}

What is the diagnosis and what is the treatment?

See page 324 for the answer

\section{K Subramaniam, ${ }^{1,2}$ H C Ee, ${ }^{2}$ S Fermoyle, ${ }^{3}$ C W Siah ${ }^{1}$}

'Department of Gastroenterology, Royal Perth Hospital, Perth, Western Australia; ${ }^{2}$ Department of Gastroenterology, Sir Charles Gairdner Hospital, Perth, Western Australia; ${ }^{3}$ Pathwest Laboratory Medicine, Royal Perth Hospital, Perth, Western Australia

Correspondence to Dr Kavitha Subramaniam, Department of Gastroenterology, Sir Charles Gairdner Hospital, Hospital Avenue, Nedlands 6009, Western Australia; kaviths@hotmail.com

\section{Competing interests None.}

Patient consent Obtained.

Provenance and peer review Not commissioned; externally peer reviewed.

Published Online First 28 September 2010

Gut 2011;60:298. doi:10.1136/gut.2010.215277 